\title{
Intramural hematoma and penetrating ulcer in the descending aorta: differences and similarities
}

\author{
Arturo Evangelista ${ }^{1,2}$, Giuliana Maldonado ${ }^{2}$, Sergio Moral ${ }^{3}$, Gisela Teixido-Tura ${ }^{1}$, Angela Lopez ${ }^{1}$, \\ Hug Cuellar ${ }^{4}$, Jose Rodriguez-Palomares ${ }^{1}$
}

${ }^{1}$ Servei de Cardiología, Hospital Universitari Vall d’Hebron, CIBER-CV, Barcelona, Spain; ${ }^{2}$ Instituto del Corazón, Quironsalud Teknon, Barcelona, Spain; ${ }^{3}$ Cardiology Department, Hospital Universitari Doctor Josep Trueta, CIBER-CV, Girona, Spain; ${ }^{4}$ Institut Diagnostic per la Imatge, Hospital Vall d'Hebron, Barcelona, Spain

Correspondence to: Arturo Evangelista, MD, PhD. Servei de Cardiologia, Hospital Universitari Vall d'Hebron, $\mathrm{P}^{\circ}$ Vall d'Hebron 119 , 08035

Barcelona, Spain. Email: arturevangelistamasip@gmail.com.

\begin{abstract}
Acute aortic syndromes include a variety of overlapping clinical and anatomic diseases. Intramural hematoma $(\mathrm{IMH})$, penetrating atherosclerotic ulcer (PAU), and aortic dissection can occur as isolated processes or can be found in association. All these entities are potentially life threatening, so prompt diagnosis and treatment is of paramount importance. IMH and PAU affect patients with atherosclerotic risk factors and are located in the descending aorta in $60-70 \%$ of cases. IMH diagnosis can be correctly made in most cases. Aortic ulcer is a morphologic entity which comprises several entities-the differential diagnosis includes PAU, focal intimal disruptions (FID) in the context of IMH evolution and ulcerated atherosclerotic plaque. The pathophysiologic mechanism, evolution and prognosis differ somewhat between these entities. However, most PAU are diagnosed incidentally outside the acute phase. Persistent pain despite medical treatment, hemodynamic instability, maximum aortic diameter (MAD) $>55 \mathrm{~mm}$, significant periaortic hemorrhage and FID in acute phase of IMH are predictors of acute-phase mortality. In these cases, TEVAR or open surgery should be considered. In non-complicated IMH or PAU, without significant aortic enlargement, strict control of cardiovascular risk factors and frequent follow-up imaging appears to be a safe management strategy.
\end{abstract}

Keywords: Intramural hematoma (IMH); aortic ulcer (AU); penetrating atherosclerotic ulcer (PAU); focal intimal disruption; acute aortic syndrome (AAS)

Submitted Jul 06, 2019. Accepted for publication Jul 16, 2019.

doi: 10.21037/acs.2019.07.05

View this article at: http://dx.doi.org/10.21037/acs.2019.07.05

\section{Introduction}

Acute aortic syndrome (AAS) is defined as an acute process in the aortic wall caused by disruption of the medial layer to a varying degree with the risk of aortic rupture and other complications (1-8). Aortic intramural hematoma (IMH) together with aortic dissection are entities included in AAS (3). While in classical aortic dissection flow communication occurs through a primary intimal tear and blood propagation creates a false lumen, in IMH, hemorrhage occurs within the aortic wall in the absence of primary intimal disruption (5). Penetrating atherosclerotic ulcer (PAU) is a primary disruption in the intimal layer of the aortic wall due to atherosclerotic plaque (9). The pathophysiologic mechanism, evolution and prognosis differs between these entities. However, some patients occasionally exhibit several or all of these lesions, thus, demonstrating the existence of a link between them. In such cases it is difficult to know which occurred first. This keynote lecture was undertaken to assess and present the differences and similarities in pathophysiology, diagnosis, evolution and management between IMH and PAU.

\section{Pathophysiology}

Krukenberg (6) first proposed that rupture of the vasa 
vasorum initiated the process of aortic dissection in 1920. Gore (1) championed this view in the 1950s and suggested that underlying media degeneration predisposed the vasa vasorum to hemorrhage and IMH. Rupture of the vasa vasorum has been related to the atherosclerotic process and systemic hypertension. A further hypothesis is that IMH originates from small entry tears in the intima followed by thrombosis of these tears, rendering them difficult to detect by imaging studies (10). Other authors have proposed intimal fracture of an atherosclerotic plaque as the primary event, which then permits propagation of blood into the aortic media. In such a chronic setting, however, the hematoma is confined to the area adjacent to an atherosclerotic ulcer (7-9). The strong relationship between intramural hemorrhage and the atherosclerotic process explains why IMH and PAU are located in the descending aorta in $60-70 \%$ of cases. IMH can also be provoked by a thoracic traumatic contusion or percutaneous catheter manipulation, as in angioplasty, insertion of a balloon pump or catheter ablation on the left side (8).

PAU was first defined by Stanson et al. in 1986 (9) as a pathologic feature characterized by ulceration in aortic atheroma which deepens through the elastic lamina into the media. However, not all aortic ulcers (AU) are penetrating atherosclerotic ulcers. "Aortic ulcer" is a global term based on morphologic findings, which includes several etiologies such as infective, inflammatory, traumatic, iatrogenic, atherosclerotic or secondary to IMH. Nevertheless, different names have been proposed for similar lesions and, moreover, the acronym of PAU has become widespread and used not only as an abbreviation of Penetrating Aortic Ulcer but also for Penetrating Atherosclerotic Ulcer.

\section{Prevalence and risk factors}

The prevalence of IMH in AAS ranges from 5\% to $27 \%$ (2,11-18), with the majority involving the descending aorta. This great disparity in the reported incidence is justified by various factors such as the referral rate of patients from community hospitals and the sensitivity of imaging techniques used by each center to diagnose small IMH. An autopsy study noted that $13 \%$ of patients with a diagnosis of aortic dissection had IMH (19). Asian groups reported a higher incidence of IMH than the International Registry of Acute Aortic Dissection (IRAD) series: 28.9\% vs. $5.7 \%(16,20,21)$. A possible explanation could be that the diagnosis of IMH is suspected more frequently in Asian than in Western hospitals. However, this low incidence of
IMH in the IRAD series may be due to the fact that IRAD centers are referral hospitals and some mild IMH may have gone undiagnosed at primary centers (22).

PAU accounts for $5 \%$ to $7.5 \%$ of all cases of AAS (23-26), and autopsy series found nearly $5 \%$ of dissections had originated from a PAU (25). In around $50 \%$ of cases, concurrent aortic aneurysms are present (7). Patients with $\mathrm{IMH}$ and PAU are older than those with classical dissection, $69 \pm 10$ vs. $62 \pm 14 \mathrm{y}$, and risk factors such as hypertension, hypercholesterolemia or smoking tend to be more frequent than in classical dissection (21).

\section{Clinical presentation}

IMH and PAU are difficult to distinguish from classical dissection on purely clinical grounds. Abrupt onset of severe chest or back pain are the most common presenting symptoms. Pain is frequently located in the back. Migration of pain is similar to that of dissection (16\%), but rarely reaches the legs: $2 \% v s .11 \%$ (21). Some patients with PAU are asymptomatic and the diagnosis is made incidentally on imaging tests performed for other reasons. Compared with classical dissection, patients with IMH and PAU seldom have aortic regurgitation, pulse deficits or lower limb ischemia. On initial examinations, patients with IMH often have a normal electrocardiogram (46\% vs. $30 \%)$ and a similar tendency to have no abnormalities on initial chest X-ray compared with classical dissection (23\% vs. $15 \%)(21)$.

\section{Diagnosis}

By definition, IMH and PAU have neither an intimal flap nor double channel intraluminal flow. Thanks to advances in non-invasive imaging modalities, such as computed tomography (CT) (27), cardiovascular magnetic resonance (CMR) $(2,28)$ and transesophageal echocardiography (TEE) (26), the IMH diagnosis can be correctly made in most cases (Figure 1). However, establishing the diagnosis requires more imaging tests than classical dissection (21). One of the IRAD findings is the low incidence of IMH in AAS, approximately $6 \%$ (21). This percentage was clearly lower than that reported by other series published from a single center $(2,10,14-18)$, and may reflect the degree to which the diagnosis was sought among patients with abnormal but non-diagnostic initial imaging studies. The incidence of PAU in the IRAD has not been reported. PAU can be found throughout the aorta but are most common 

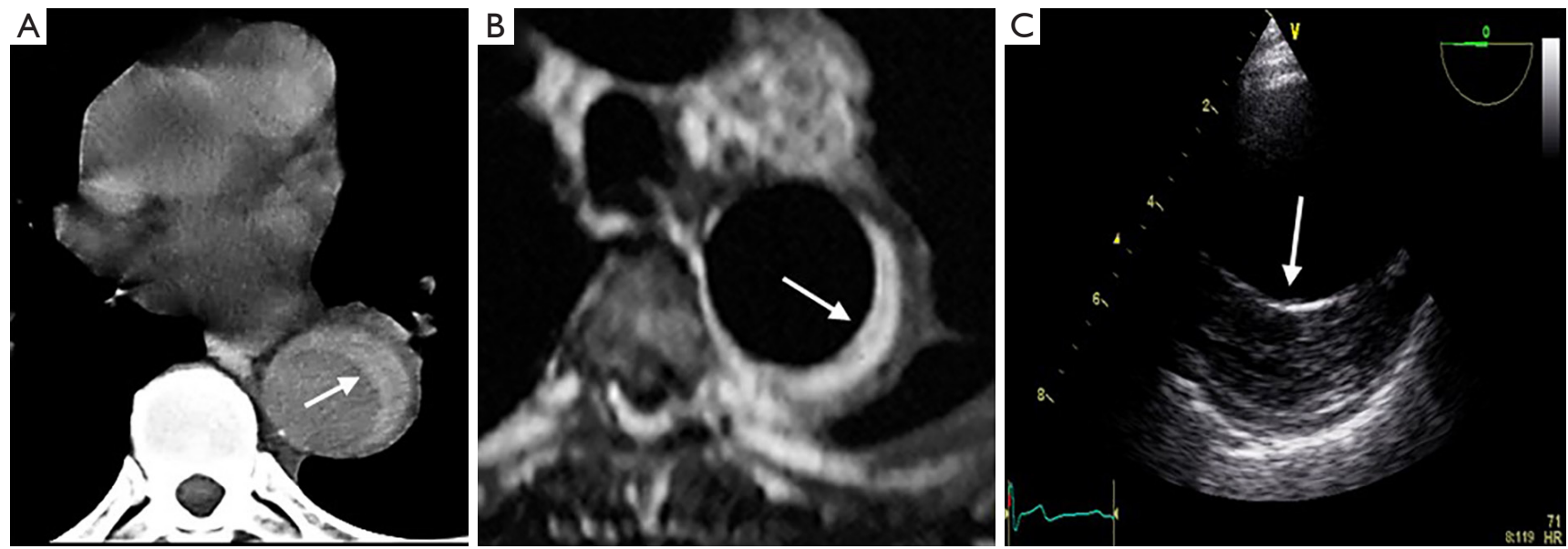

Figure 1 Acute type B IMH. (A) CT is useful in early diagnosis, particularly, if we do not use contrast (arrow shows this high attenuation area secondary to a recent bleed); (B) CMR facilitates diagnosis by the hyperintense imaging in T2 (arrow); (C) TEE shows he intramural hematoma with mild calcification of the intima (arrow). IMH, intramural hematoma; CMR, cardiovascular magnetic resonance; TEE, transesophageal echocardiography

in the descending thoracic aorta. Nathan et al. reported the largest series to date of $388 \mathrm{PAU}$ in 315 patients (29). The ulcer was located in the descending thoracic aorta in $62 \%$, abdominal aorta in $31 \%$ and aortic arch in $7 \%$.

\section{Imaging technique findings}

CT is the most used imaging technique in the diagnosis of AAS (30). IMH is diagnosed on CT by a high attenuation area, usually crescentic or circular, which fails to enhance after injection of contrast medium (27) (Figure 1A). Crescentic aortic wall thickening without intimal flap is very easily detected by CMR; the signal intensity of the thickened aorta is secondary to methemoglobin formation within the hematoma, resulting in increased signal intensity on T1-weighted images in subacute IMH (2) (Figure 1B). TEE is also useful to demonstrate thickening of the aortic wall $(26,27)$ (Figure 1C) although it offers no signals to identify acute versus chronic hematoma, and a differential diagnosis with other entities that also demonstrate aortic wall thickening, such as severe atherosclerosis of the intima or aortitis, should be made.

Normal aortic wall thickness is less than $3-4 \mathrm{~mm}$ using any imaging modality, hence, aortic wall thickness greater than $5 \mathrm{~mm}$ with typical clinical symptoms suggesting AAS suggests a diagnosis of IMH. One characteristic finding of IMH is the presence of echolucent areas or echo-free space within the thickened aorta wall (31). The challenge of early $\mathrm{IMH}$ diagnosis is considerable. Given the dynamic evolution of IMH and its the difficulty in making a diagnosis when the $\mathrm{IMH}$ is small, multiple diagnostic imaging tests are needed to confirm its presence (21). CT with contrast enhancement makes it possible to demonstrate, in selected patients, a small communication through an intimal micro-tear, but this is exceptional in the acute phase. TEE with color Doppler may be superior to any other technique for assessing the intima and demonstrating small communications (Figure 2). However, the wide field of vision of CT and CMR is important to correctly define the extension of IMH and periaortic bleeding. The presence of fluid extravasation, pericardial and pleural effusion and mediastinal hemorrhage are frequent findings in IMH $(14,18)$.

The typical PAU is a contrast-filled, pouch-like protrusion of the aorta in the thickened aortic wall in an atherosclerotic process (Figure 3). Often, extensive atherosclerosis, apart from ulceration, is also found. TEE demonstrates a localized crater-like protrusion of the aortic lumen below or beside an atherosclerotic plaque without intimal flap and false lumen (26) (Figure 4).

\section{Differential diagnosis}

IMH is easily differentiated from classical aortic dissection; an intimal tear or flap are absent in the acute phase and there is no evidence of direct flow communication. However, the diagnosis can be very difficult when the false lumen of the dissection is totally thrombosed. In this case, only identification of an entry tear, during surgery or 

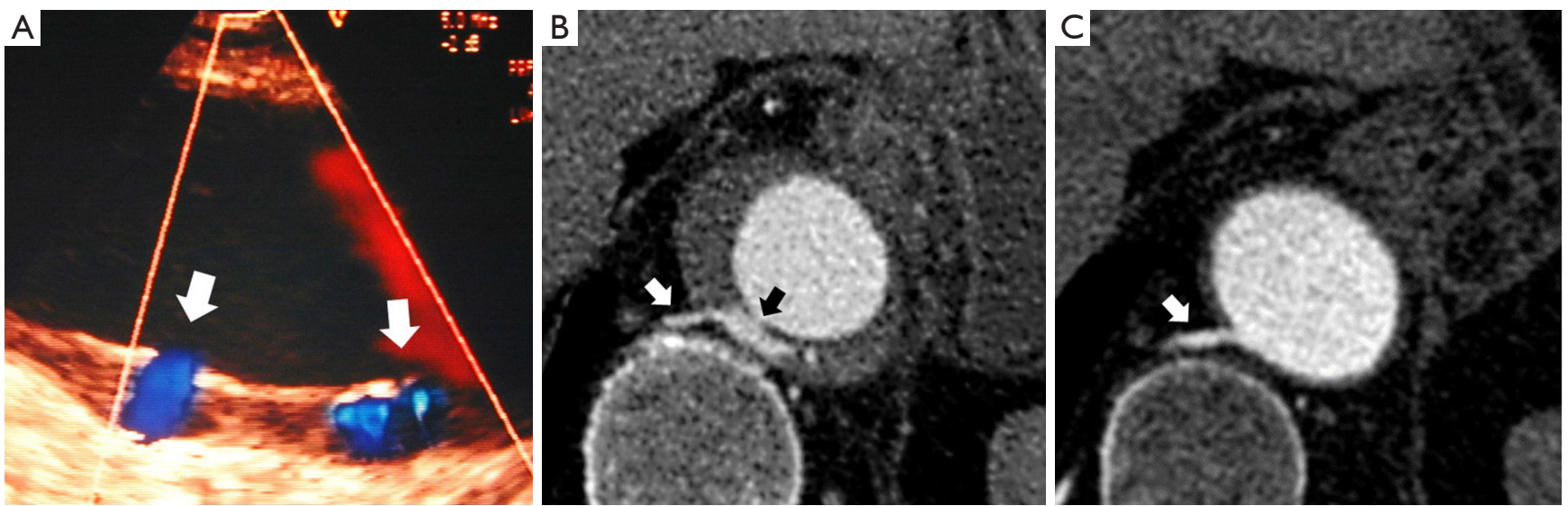

Figure 2 Tiny intimal disruptions. (A) TEE with color Doppler may be superior to any other technique for assessing the intima and demonstrating small communications (arrows) usually associated with intercostal or lumbar artery ostia; (B,C) contrast CT enhancement demonstrating a tiny intimal disruption through intimal micro-tear (black arrow), associated with an artery branch (white arrows), which disappears with IMH reabsorption. TEE, transesophageal echocardiography; IMH, intramural hematoma.
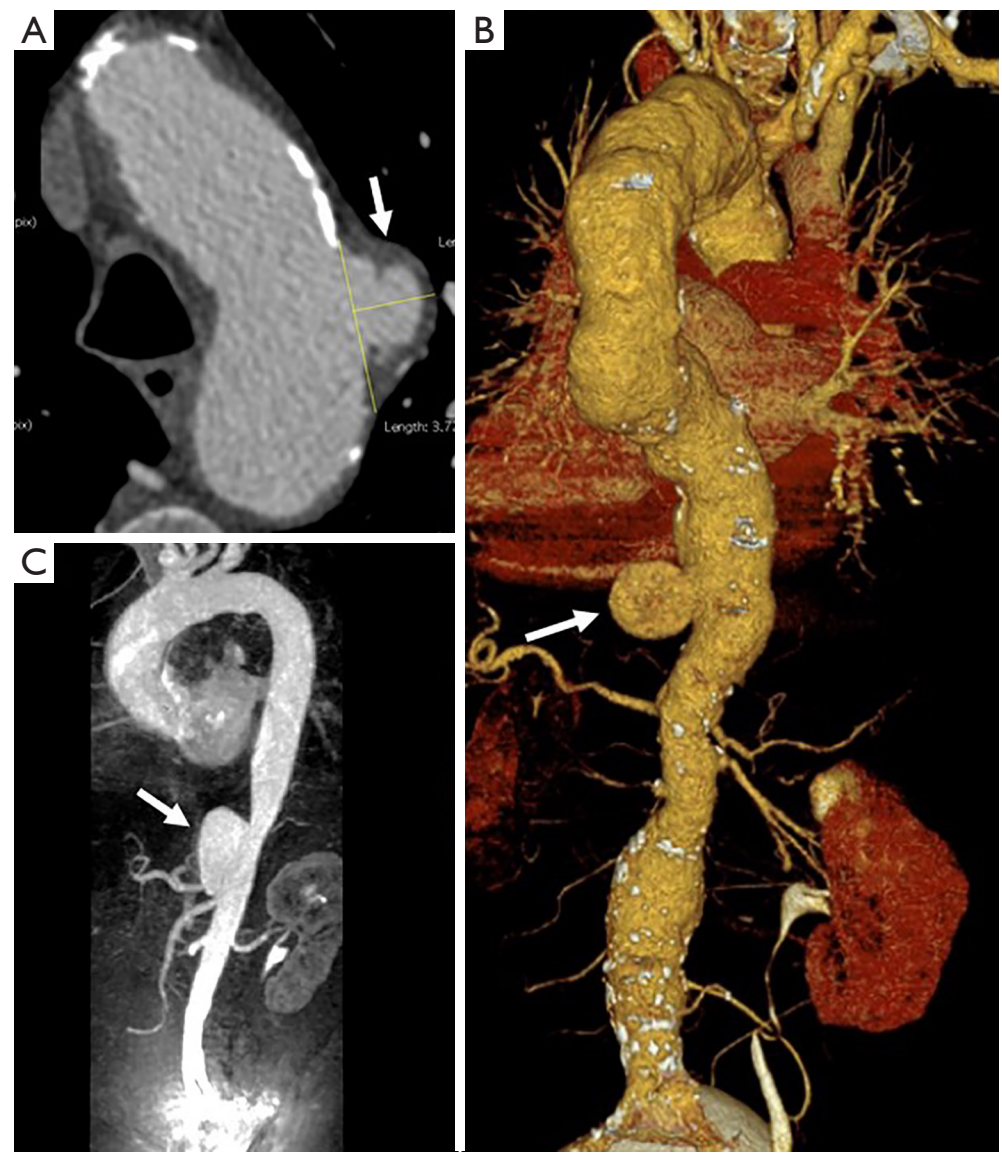

Figure 3 Aortic ulcers by CT: (A) penetrating atherosclerotic ulcer (arrow) in a calcified aortic arch; (B) focal intimal disruption with localized dissection (arrow); (C) angiotomography with multiplanar reconstruction (MPR) showing a large PAU in distal thoracic aorta (arrow). PAU, penetrating atherosclerotic ulcer. 

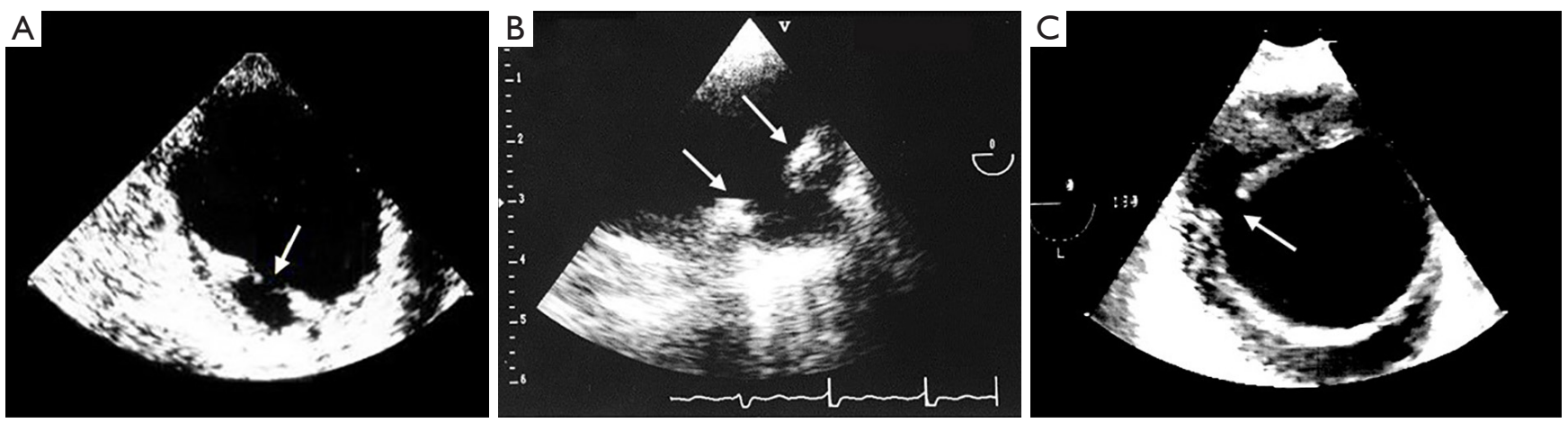

Figure 4 Usefulness of TEE in the differential diagnosis of aortic ulcer types: (A) ulcerated plaque in descending thoracic aorta intima without involvement of the media layer of aortic wall (arrow); (B) atherosclerotic ulcerated lesion penetrating through the aortic intima into the aortic wall that progresses to the tunica media (arrows) and is diagnosed by a pouch-like protrusion into the aortic wall; (C) intramural hematoma evolution showing a localized dissection from a focal intimal disruption (FID) with an orifice diameter of 4 mm (arrow). TEE, transesophageal echocardiography.

autopsy, permits correct differentiation. On the other hand, only complete chronologic imaging findings can provide a correct differential diagnosis between aortic dissection with total thrombus of the proximal or distal part versus an IMH which evolves to classical dissection in one of the segments.

One challenging differential diagnosis of IMH is aortic wall thickening caused by atherosclerotic changes or aneurysmal dilation with mural thrombi (31). When distinguishing IMH from other aortic conditions, identification of the intima and careful observation of the inner surface of the thickened aortic wall are helpful. Usually, the inner margin of an IMH is smooth and aortic thickening occurs beneath the bright echo-dense intima, whereas an irregular margin caused by thickening above the intima with a dilated aorta is commonly observed in patients with aneurysmal dilation and mural thrombi. In this respect, the presence of intimal calcium can often be used to distinguish an intramural hematoma from an intraluminal thrombus.

As discussed previously, $\mathrm{AU}$ is a morphologic term of imaging techniques which includes several entities and requires a differential diagnosis among PAU, focal intimal disruptions (FID) with ulcer-like projections (ULP) in the context of IMH evolution, and ulcerated atherosclerotic plaque (Figure 4). In the aortic atherosclerotic classification by TEE, the highest risk of emboli is present in cases with ulcerated or mobile plaques (32). These lesions have irregular margins, are located in the intimal layer and are not associated with contrast material extension beyond the calcified intima assessed by CT or TEE. By contrast, PAU is an ulcerated lesion penetrating through the aortic intima into the aortic wall that progresses to the tunica media and is diagnosed by a pouch-like protrusion into the aortic wall by imaging techniques $(7,9,29)$. PAU lesions in the acute phase are frequently accompanied by a hematoma localized around the lesion. In addition, two types of intimal disruption have been observed during the course of an IMH: FID and tiny intimal disruptions (TID) (33). FID is defined as a focal, contrast materialfilled pouch projecting outside the opacified aortic lumen with an orifice diameter $>3 \mathrm{~mm}$. TID with orifice diameter $\leq 3 \mathrm{~mm}$ are stable images usually associated with intercostal or lumbar artery ostia (34-36). By contrast, an intramural blood pool is a similarly small, localized area of contrast enhancement within the IMH, however, it has a very small $(<2 \mathrm{~mm})$ or imperceptible communication with the aortic lumen $(26,27,37)$. Patients with a thicker IMH are more likely to develop focal contrast enhancement. TID are more likely to occur in the descending aorta and are sometimes referred to as an aortic branch artery tear or an aortic branch artery pseudoaneurysm since there often is a visible connection with an intercostal, lumbar or bronchial artery (Figure 2) $(35,36)$. Although TEE has less sensitivity than $\mathrm{CT}$ in the diagnosis of $\mathrm{AU}$, it is the technique of choice in the differential diagnosis of these entities, as illustrated in Figure 5. One of the most challenging scenarios of AU for the differential diagnosis, but also for prognosis and treatment implications, is when they are related to an AAS. This association mainly implies two etiopathologies: atherosclerotic $(\mathrm{PAU})$, and intimal disruption secondary to 

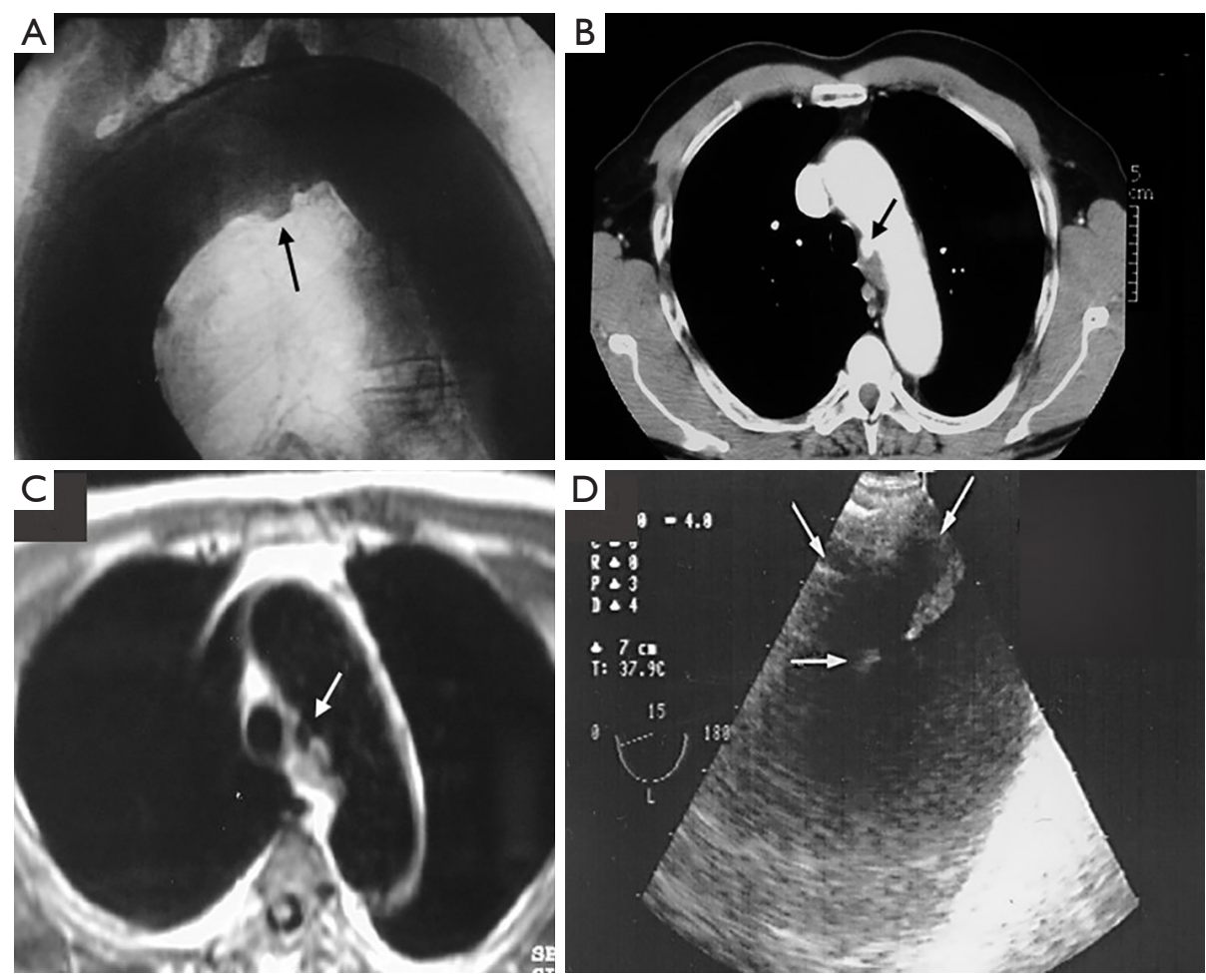

Figure 5 Penetrating aortic ulcer located in the aortic arch (arrows) diagnosed by different imaging techniques: aortography (A); angio CT (B); CMR (C) and TEE (D). Only TEE identified the localized dissection (FID) from the natural evolution of intramural hematoma. CMR, cardiovascular magnetic resonance; TEE, transesophageal echocardiography; FID, focal intimal disruption.

IMH with FID. A differential diagnosis of the two entities is not always possible owing to their rapid morphologic evolution. Thus, depending on exactly when an imaging 'snapshot' is taken after symptoms onset, an FID may be erroneously interpreted. Furthermore, the behaviour of FID in acute or subacute/chronic phase of IMH, as well as their pathophysiology, are also different and in many cases change the recommended treatment (35). The differential diagnosis between PAU and FID is shown in Table 1.

\section{Early evolution, complications and risk stratification}

Prospective studies revealed evidence that IMH eventually either reabsorbs, or progresses to classical dissection, contained rupture or formation of an aneurysm within 30 days of hospital admission $(14,15,21,33)$. Acute mortality in the overall published series of more than 500 cases has been considered to be $14 \%$ in type B IMH $(11,38)$. In our series, mortality in the first three months of evolution was $12 \%$ (14). Patient mortality with maximum aortic diameter
(MAD) over $50 \mathrm{~mm}$ was $50 \%$ and only $2 \%$ in those with diameter less than $50 \mathrm{~mm}$ (14).

Sueyoshi et al. (39) reported that MAD over $40 \mathrm{~mm}$ predicted IMH progression. A possible explanation for the prognostic value of MAD in short-term IMH evolution is that when intramural bleeding weakens the aortic wall, the greater stress on the dilated aortic wall implies a greater risk of rupture than that of the non-dilated aorta.

Several published series showed periaortic bleeding to be more frequent in IMH than in classical dissection $(14,18)$. Although this bleeding is not necessarily the equivalent of aortic rupture and most IMH tend to regress without complications (Figure 6), mortality in our patients with this complication was high (35-43\%), particularly in the subgroup of cases who presented acute anemia on blood tests (14). However, progressive accumulation of a large amount of pleural effusion is not in itself an indication for surgical treatment.

Risk stratification based on clinical and diagnostic imaging findings in patients with type B IMH is given in Table 2. Most of these predictors may be defined by 


\begin{tabular}{|c|c|c|c|}
\hline \multirow{2}{*}{ Characteristics } & \multirow{2}{*}{ PAU (acute) } & \multicolumn{2}{|l|}{ FID } \\
\hline & & Acute & Subacute/chronic \\
\hline Incidence in type B IMH & $\begin{array}{l}\text { Very infrequent; probable } 2-3 \% \\
\text { of type B IMH }\end{array}$ & Infrequent; $10 \%$ of type B IMH & Frequent; $30 \%$ of type B IMH \\
\hline Pathophysiology & Atherosclerosis & Expansion of IMH & Regression of IMH \\
\hline $\begin{array}{l}\text { Diagnosis timing with } \\
\text { imaging techniques }\end{array}$ & Lesion present in the first study & $\begin{array}{l}\text { Development during the first } 14 \text { days } \\
\text { after symptom onset (infrequent in } \\
\text { the first study) }\end{array}$ & $\begin{array}{l}\text { Development between } 2 \text { weeks } \\
\text { and } 6 \text { months after symptom onset }\end{array}$ \\
\hline \multicolumn{4}{|c|}{ Morphological characteristics of lesions } \\
\hline \multirow{2}{*}{$\begin{array}{l}\text { Definition and main } \\
\text { characteristics of the } \\
\text { lesion for diagnosis }\end{array}$} & $\begin{array}{l}\text { 2. Present from the beginning: } \\
\text { cause of IMH and AAS }\end{array}$ & $\begin{array}{l}\text { 2. Communicating orifice with aortic } \\
\text { lumen }>3 \mathrm{~mm}\end{array}$ & $\begin{array}{l}\text { 2. Communicating orifice with } \\
\text { aortic lumen }>3 \mathrm{~mm} \text {. Often has a } \\
\text { small intimal flap }\end{array}$ \\
\hline & & $\begin{array}{l}\text { 3. Development within the first } \\
14 \text { days after symptom onset in the } \\
\text { absence of new acute episodes or } \\
\text { re-bleeding }\end{array}$ & $\begin{array}{l}\text { 3. Development after the first } \\
14 \text { days of symptom onset in the } \\
\text { absence of new acute episodes or } \\
\text { re-bleeding }\end{array}$ \\
\hline
\end{tabular}

$\mathrm{IMH}$, intramural hematoma; FID, focal intimal disruption; PAU, penetrating atherosclerotic ulcer.

imaging techniques (40): (I) MAD in the acute phase is one of the major predictors of progression in type B IMH $(14,39,43)$. Patients with a MAD $>45 \mathrm{~mm}$ have a higher risk of dissection, regardless of the location $(14,41)$; (II) wall thickness has been described as a predictor of progression $(14,41,42,44)$; however, this issue is controversial (45). Sueyoshi et al. (44) proposed a cut-off $\geq 10 \mathrm{~mm}$, although this value varied considerably from 10 to $15 \mathrm{~mm}$, in the different series published; (III) the incidence of periaortic hemorrhage or pleural effusion is higher in IMH than in aortic dissection; in some studies, this incidence rose to $40 \%$ (45). In some series, pleural effusion led to an unfavorable prognosis for IMH $(42,45)$. However, at least two mechanisms may explain this finding: leakage of blood from the aorta through microperforations or a nonhemorrhagic exudate from an aortic wall inflammatory reaction owing to the proximity of the IMH to the adventitia; (IV) FID is a frequent finding in type B IMH, and its incidence ranges from $20-60 \%$ of cases $(33,46,47)$. In most cases, FIDs result from a localized dissection. The prognostic significance of FID initiated in the subacute or chronic phase is unclear, and discrepancy exists as to its real meaning in the context of type B IMH. This specific complication will be discussed in the treatment section. 

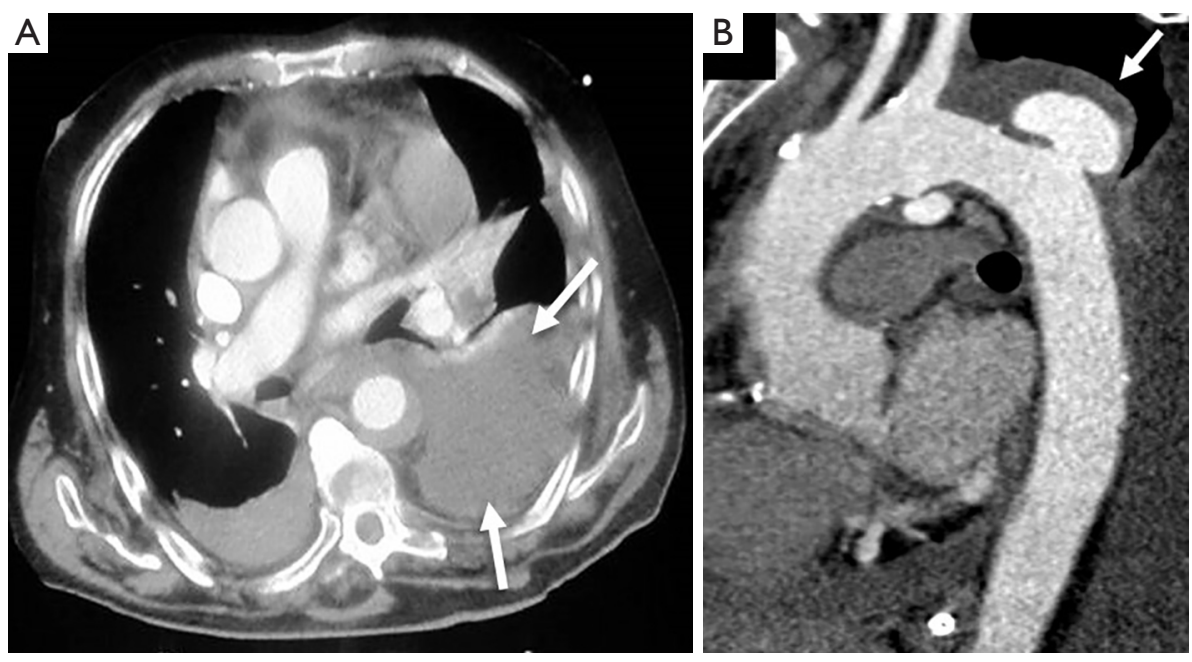

Figure 6 Complicated intramural hematoma in acute phase. (A) Periaortic hematoma with large hemothorax (arrows); (B) FID with a large pseudoaneurysms (ulcer-like projection) (arrow). FID, focal intimal disruption.

\begin{tabular}{ll}
$\begin{array}{l}\text { Table } 2 \text { Significant predictors of aortic complications in acute type } \\
\text { B IMH }\end{array}$ & $\begin{array}{l}\text { Cut-off or sign of } \\
\text { complicated evolution }\end{array}$ \\
\hline High-risk feature & $>45 \mathrm{~mm}(14,40)$ \\
\hline Maximum aortic diameter & $\geq 10 \mathrm{~mm}(41)$ \\
Wall thickness of involved segment & Presence $(14,42)$ \\
Periaortic hemorrhage & Presence $(42,43)$ \\
\hline Pleural effusion & Acute $(30)$ \\
FID & $>3 \mathrm{~mm}(30)$ \\
\hline Time presentation &
\end{tabular}

FID, focal intimal disruption; IMH, intramural hematoma.

Moral et al. (33) showed that FID development in the acute phase of type B IMH has poor prognosis owing to the high risk of aortic rupture. In our experience, most FID diagnosed in the subacute or chronic phase evolve with slow aortic dilation and without complications (48) (Figure 7). On the basis of the current available literature, TID does not appear to pose an increased risk for IMH progression, need for surgery, or mortality but does imply a higher risk for incomplete hematoma reabsorption $(38,49)$. Larger TID and those with a visible connection to a branch artery are at higher risk for incomplete reabsorption and may grow over time, necessitating endovascular embolization (50).
The natural history of PAU is a matter of ongoing debate. When first described, PAU was considered a malignant entity with a high risk of rupture or progression to frank dissection. However, subsequent studies suggested a more benign course with watchful waiting advocated by several groups $(51,52)$. This controversy is exemplified by the respectfully opposing views expressed by investigators at Yale University (53) and Mayo Clinic (54). A recent analysis of clinical outcomes of $26 \mathrm{PAU}$ patients from the Yale University Thoracic Aortic Database supported an aggressive surgical approach (53). One-third of their patients presented with rupture, and two-thirds underwent surgery during their index hospitalization. In contrast, a subsequent study from the Mayo Clinic demonstrated a much less aggressive course, with only 29 of 105 patients with PAU undergoing surgery, and no difference in late outcome for medical versus surgical groups (54). Spontaneous rupture of the aorta in PAU is a rare condition in the absence of AAS or severe progressive dilation. However, in symptomatic patients, the risk of complications may be high. Significant predictors of aortic rupture (Table 3) are considered to be recurrent or refractory pain despite medical treatment $(7,9)$, hemodynamic instability, periaortic bleeding or significant/ progressive pleural effusion, association with IMH, and large ulcer size $(23,43)$.

\section{Acute treatment}

Acute IMH involving the descending aorta has an in- 


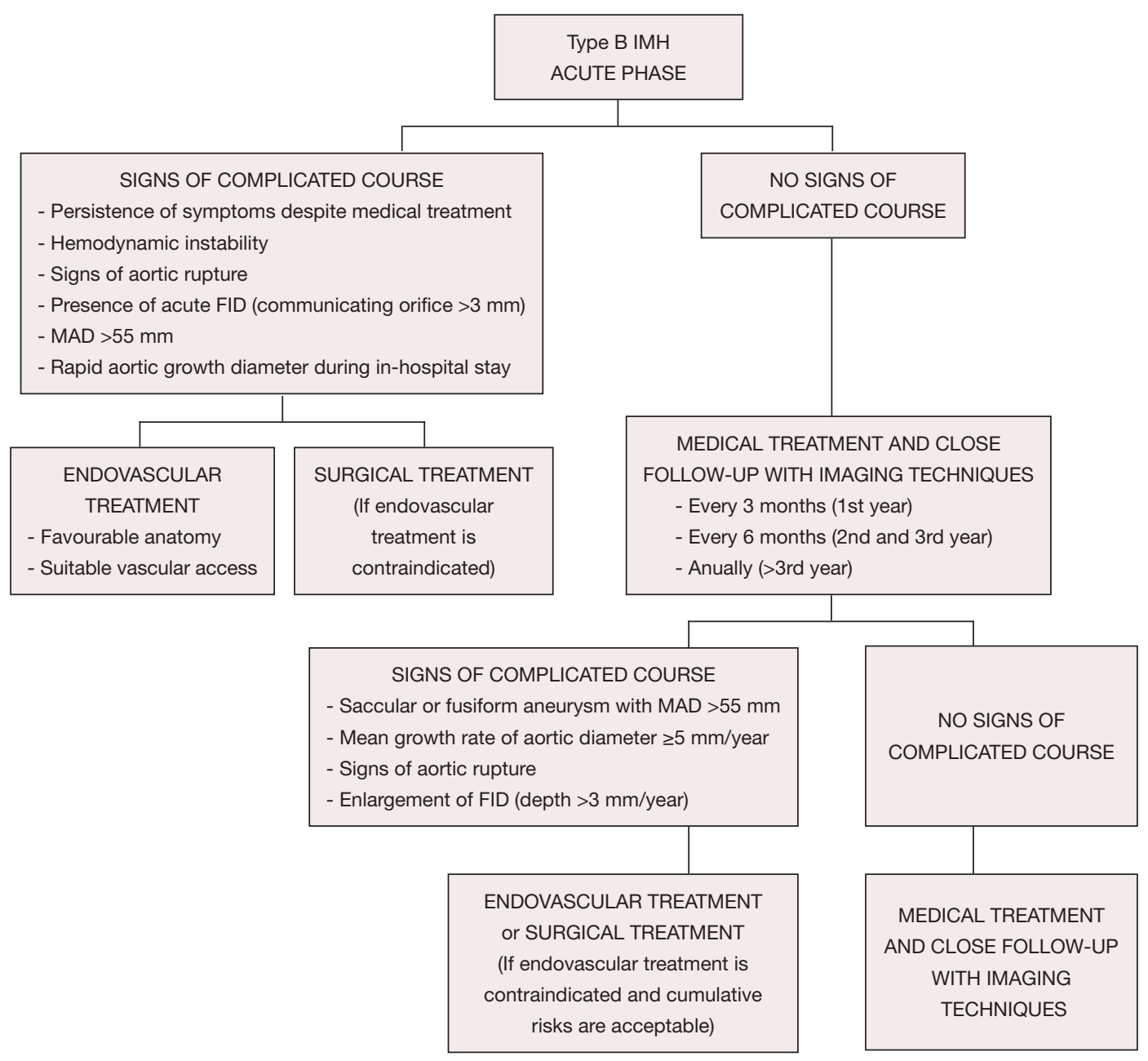

Figure 7 Acute and chronic management pathway for type B IMH. FID, focal intimal disruption; MAD, maximum aortic diameter; IMH, intramural hematoma.

Table 3 High-risk features of aortic complications in type B PAU

\begin{tabular}{|c|c|}
\hline High-risk feature & $\begin{array}{l}\text { Cut-off or sign of complicated } \\
\text { evolution }\end{array}$ \\
\hline Symptomatic patient & $\begin{array}{l}\text { Symptoms despite medical treatment } \\
\text { or haemodynamic instability }(7,9,23)\end{array}$ \\
\hline \multicolumn{2}{|l|}{ Asymptomatic patient } \\
\hline Periaortic bleeding & Presence $(23,39)$ \\
\hline Pleural effusion & Significant/progressive $(23,39)$ \\
\hline IMH-associated & Presence of IMH $(23,39)$ \\
\hline Initial PAU size & $\begin{array}{l}\text { Large initial depth or high growth rate } \\
\text { size }(23,39)\end{array}$ \\
\hline $\begin{array}{l}\mathrm{IMH} \text {, intramural hem } \\
\text { ulcer. }\end{array}$ & ma; PAU, penetrating atherosclerotic \\
\hline
\end{tabular}

hospital mortality risk of less than $10 \%$, similar to that with descending or type B aortic dissection. The literature supports the use of medical therapy as the initial treatment for this condition. Acute IMH confined to the arch remains a controversial issue. In the IRAD series, most patients were managed medically without mortality (21). Surgical treatment would have the same indications as type B aortic dissection. Nowadays, endovascular therapy can be considered, particularly in ulcer formation with rapid expansion. However, the main limitation of TEVAR in the acute phase is the high risk of secondary endoleak, intimal ruptures or pseudoaneurysm formation. These issues occur secondary to mechanical stress and the pulsatile force acting on the stent ends when the ends of the device are placed on the aortic wall affected by the IMH $(55,56)$. Sufficient 


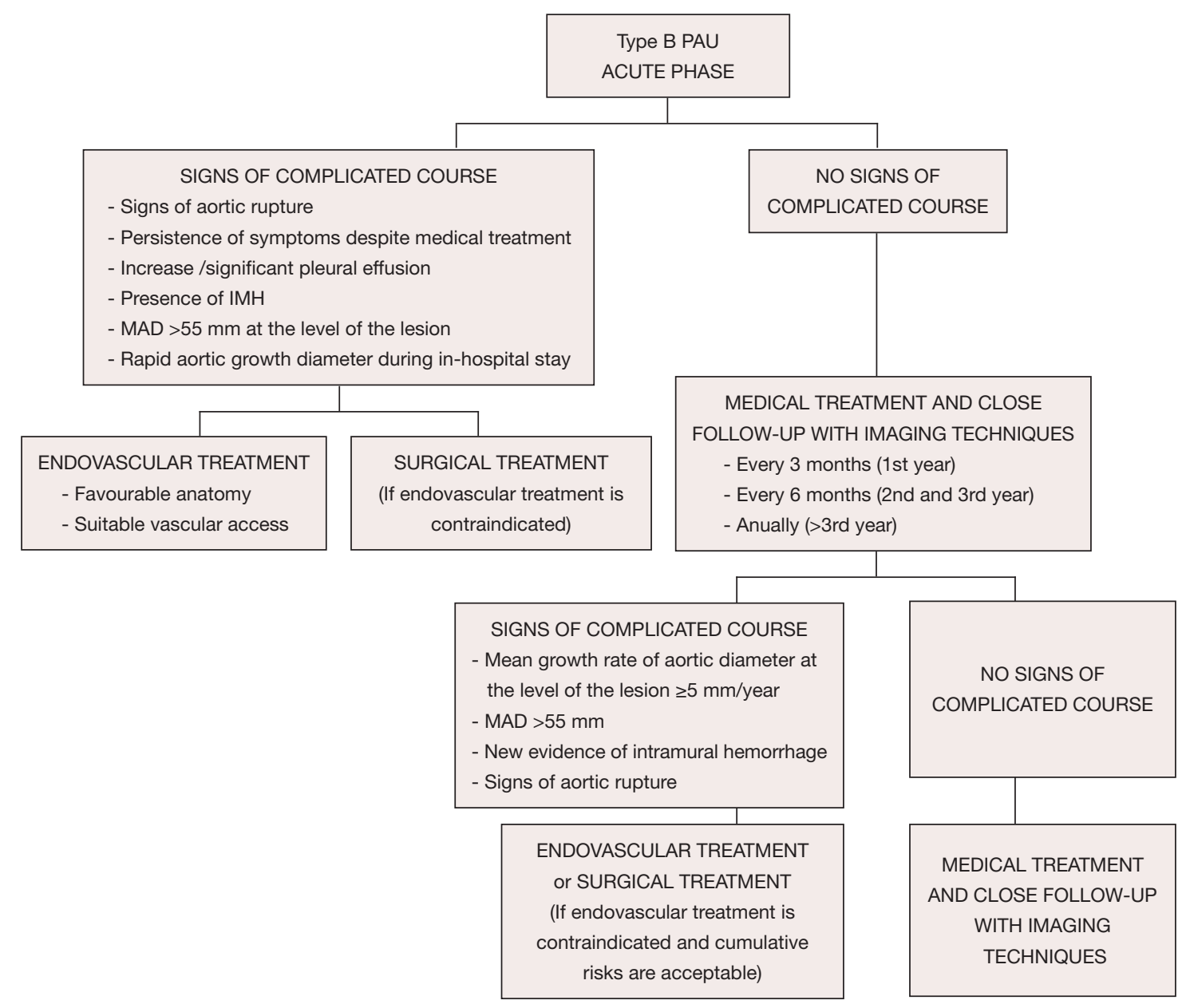

Figure 8 Acute and chronic management pathway for type B PAU. IMH, intramural hematoma; PAU, penetrating atherosclerotic ulcer.

landing zones, a minimum of $15 \mathrm{~mm}$ from the affected zone, are necessary, and in some cases, a considerable portion of the proximal and distal aorta needs to be covered.

From the acute phase, the intramural hemorrhage evolves with fibrotic changes and the aortic wall becomes more stable after 2 months. Over $40 \%$ of cases can show complete regression in the first 6 months; however, a high percentage of the remainder can evolve to classical or localized dissection and aortic aneurysm. Medical treatment and close follow-up with imaging techniques every 3 months is recommended in patients with IMH and FID without persistent pain or signs of aortic rupture in the first year (Figure 8). Thus, when FID size remains stable, followup can be made every 6 months and annually beyond the third year. Invasive treatment of IMH is indicated if MAD $>55 \mathrm{~mm}$ or the mean growth rate is $\geq 5 \mathrm{~mm} /$ year. Initial FID depth $>10 \mathrm{~mm}$ requires close follow-up and, despite of the lack of published series, values $>15 \mathrm{~mm}$ could be an indication for endovascular treatment. This latter indication necessitates the measurements being repeated using the same imaging technique, at the same aortic level, with side-by-side comparison. Treatment by TEVAR or open surgery should be based on the anatomical features of the lesion, patient comorbidities, anatomical constraints related to endograft technology and experience and results of the center in both therapeutic strategies.

As PAU is commonly observed as a segmental localized wall lesion, it is an ideal target for endovascular stent grafting. Early mortality in TEVAR treatment is estimated to be $7.2 \%$ (23). The presence of an associated IMH may increase the risk of treatment failure, aortic rupture or aorta-related death (57), thereby highlighting the need for careful planning, prudent balancing of the benefits of a possibly delayed treatment to avoid fragility of the 

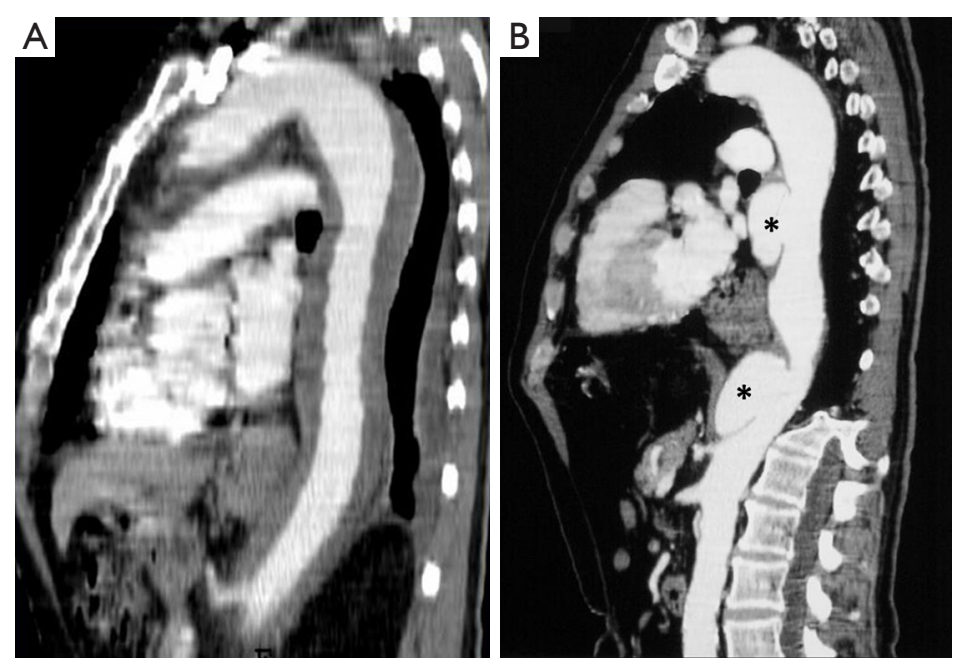

Figure 9 Initial type B IMH (A) evolving with localized dissections (asterisks) at 8 months of follow-up (B). IMH, intramural hematoma.

affected aortic wall, and other complications such as leaks and strokes. The mortality rate in the acute phase of open surgical treatment was $15.9 \%$ vs. $7.2 \%$ for TEVAR (23). Considering that many patients are inoperable or have a prohibitively high open surgery risk, TEVAR may be an excellent therapeutic option (58).

When an AU shows a mean growth rate $\geq 5 \mathrm{~mm} /$ year or MAD >55 mm, TEVAR should be considered (Figure 9); however, it is imperative to balance the risks and benefits of TEVAR in relation to age and possible comorbidities. In cases without surgical treatment criteria, follow-up every 6 months with imaging techniques for the first 3 years and every year thereafter is recommended. Asymptomatic PAU patients with ulcer diameters $>20 \mathrm{~mm}$ and/or ulcer depth $>20 \mathrm{~mm}$ have a high risk of disease progression and should be evaluated as possible candidates for early endovascular or surgical repair.

\section{Long-term evolution, complications and treatment}

Long-term prognosis of patients with type B IMH is better than in patients with aortic dissection. However, survival at 5 years reported in different series ranges from $43 \%$ to $90 \%$, depending on the population characteristics $(48,59)$. Several studies have shown significant dynamic changes in IMH during evolution, mainly during the first 6 months (59). Regression occurred within 6 months in $30 \%$ of cases, progression to dissection in $40 \%$, to classical dissection in $12 \%$, to focal dissection in $28 \%$ and $30 \%$ reabsorbed with aortic dilation (59). However, the most frequent morphologic long-term evolution of IMH is to aortic aneurysm or pseudoaneurysm formation (54\% of cases) (Figure 10).

Kaji et al. (59) showed older age and appearance of FID to be predictive of progression with type B IMH. The literature seems to confirm that one of the leading complications of IMH is localized dissection which is identified as an ulcer-like feature on imaging. Other variables such as echolucency (31) and IMH extension predict progression to aortic dissection. Song et al. (60) found no prognostic significance between echo-free space detected in IMH and progression to dissection. In that study, only TEE was performed during a short follow-up period and some localized dissections therefore may have gone unnoticed.

Spontaneous rupture of the aorta in PAU is rare in the absence of AAS or severe progressive dilation. PAU in asymptomatic patients are usually stable or have slow progression in size. No consensus exists on ulcer size cutoff values; however, growth rate and MAD at the site of the lesion have been considered, as in other aortic entities.

The long-term management of patients with successful initial treatment of IMH and PAU begins with aggressive antihypertensive therapy and maximum control of the other risk factors. Treatment with effective $\beta$-adrenergic blocking agents protects the aorta by reducing both systolic pressure and $\mathrm{dp} / \mathrm{dt}$. Progressive uptitration of dosage is advisable to achieve blood pressure $<135 / 80 \mathrm{mmHg}$. IMH and PAU should be treated with $\beta$-blockers and closely followed up 

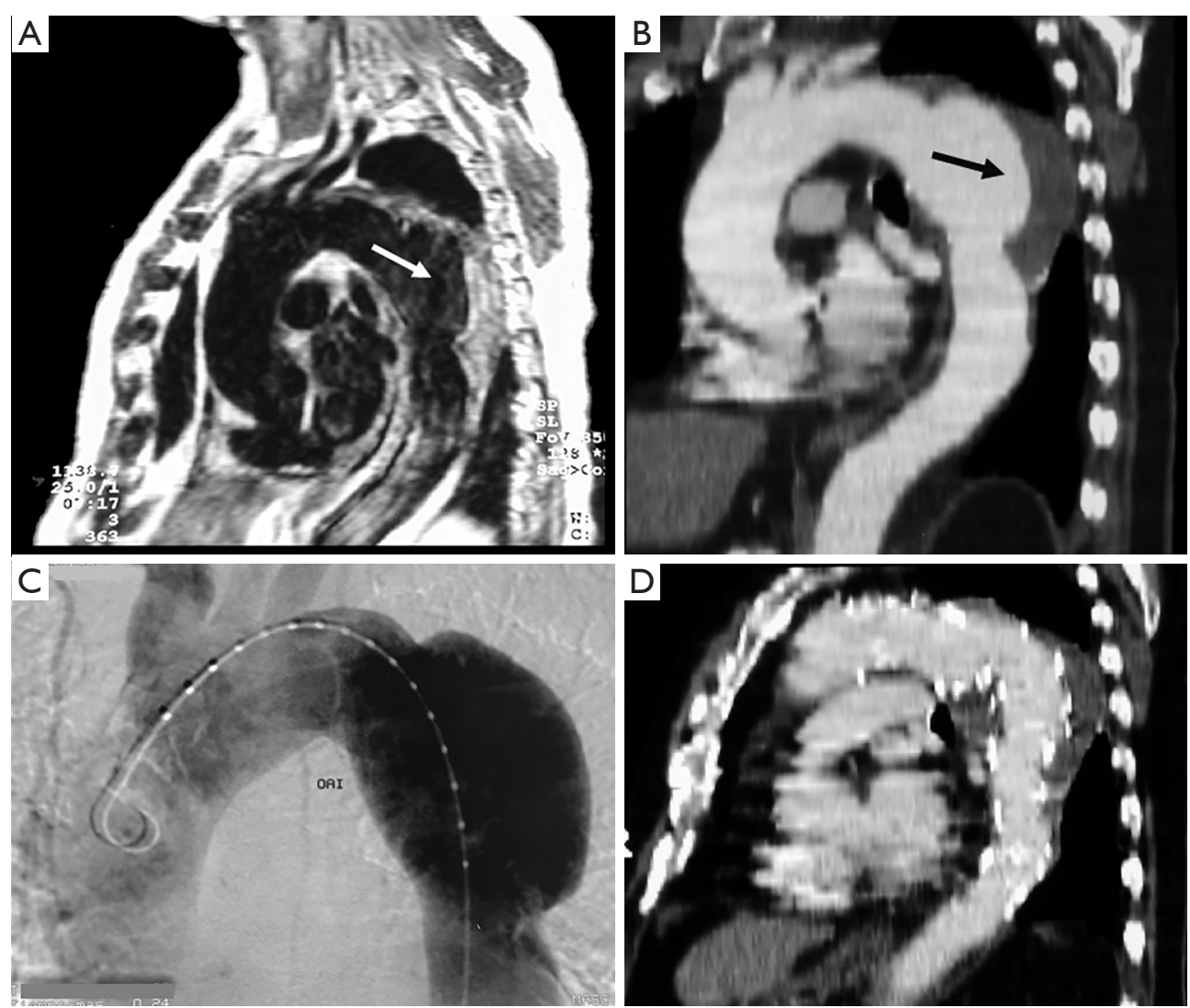

Figure 10 Evolution and management of type B IMH. (A) IMH in acute phase diagnosed by CMR (arrow); (B) significant enlargement of descending thoracic aorta during the first week of admission (arrow); (C) angiography during endovascular treatment; (D) control by angio CT one week after TEVAR procedure. IMH, intramural hematoma; CMR, cardiovascular magnetic resonance.

with imaging techniques, until complication-free absorption of the hematoma is observed and individualized thereafter. Serial imaging of the aorta is an essential component of long-term treatment. Recommendations suggest followup imaging and examination at 1, 3, 6, and 12 months after discharge and annually thereafter. The subgroup of patients with aortic dilation or subacute/chronic FID (ulcer-like images secondary to IMH) should be followed up and treated more aggressively if progressive aortic dilation is observed. Indications for surgical intervention could be (I) MAD of the affected segment $>55 \mathrm{~mm}$; (II) rapid enlargement of the affected aorta; (III) rapid enlargement of an FID; and (IV) rupture of the affected aorta. Open repair requiring graft interposition is associated with high morbidity and mortality rates, especially in patients of advanced age or with comorbidities. Endovascular placement of the stent grafts to cover the ulcer and part of the IMH has been recently investigated with promising initial results; however, this remains to be confirmed by results from large clinical series.

\section{Conclusions}

IMH and PAU in the descending thoracic aorta have significant differences in pathophysiology, evolution and management. Clinical presentation may be identical to that of classical dissection. However, most PAU are diagnosed incidentally outside the acute phase. IMH and PAU affect patients with atherosclerotic risk factors and are located in the descending aorta in $60-70 \%$ of cases. Persistent pain despite medical treatment, hemodynamic instability, MAD $>55 \mathrm{~mm}$, significant periaortic hemorrhage and FID in acute phase of IMH are predictors of acute-phase mortality. In these cases, TEVAR or open surgery should be considered. In non-complicated IMH or PAU, without significant aortic enlargement, strict control of cardiovascular risk factors 
appears to be a safe strategy; however, frequent followup imaging evaluation is required and a low threshold for intervention should be maintained if symptoms recur or if the aorta enlarges significantly.

\section{Acknowledgments}

The authors thank Christine O'Hara for help with English language review.

\section{Footnote}

Conflicts of Interest: The authors have no conflicts of interest to declare.

\section{References}

1. Gore I. Pathogenesis of dissecting aneurysm of the aorta. AMA Arch Pathol 1952;53:142-53.

2. Nienaber CA, von Kodolitsch Y, Petersen B, et al. Intramural hemorrhage of the thoracic aorta: diagnostic and therapeutic implications. Circulation 1995;92:1465-72.

3. Vilacosta I, San Roman JA. Acute aortic syndrome. Heart 2001;85:365-8.

4. O'Gara PT, DeSanctis RW. Acute aortic dissection and its variants. Circulation 1995;92:1376-8.

5. Nienaber CA, Sievers HH. Intramural hematoma in acute aortic syndrome - More than one variant of dissection? Circulation 2002;106:284-5.

6. Krukenberg E. Beitrage zur Frage des Aneurisms dissecans. Beitr Pathol Anat Allg Pathol 1920;67:329-51.

7. Coady MA, Rizzo JA, Elefteriades JA. Pathological variants of thoracic aortic dissections: penetrating atherosclerotic ulcers and intramural hematomas. Cardiol Clin 1999;17:637-57.

8. Nienaber CA, Eagle KA. Aortic dissection: new frontiers in diagnosis and management. Part I: from etiology to diagnostic strategies. Circulation 2003;108:628-35.

9. Stanson AW, Kazmier FJ, Hollier LH, et al. Penetrating atherosclerotic ulcers of the thoracic aorta: natural history and clinicopathologic correlations. Ann Vasc Surg 1986;1:15-23.

10. Chin AS, Willemink MJ, Kino A, et al. Acute Limited Intimal Tears of the Thoracic Aorta. J Am Coll Cardiol 2018;71:2773-85.

11. Maraj R, Rerkpattanapipat P, Jacobs LE, et al. Metaanalysis of 143 reported cases of aortic intramural hematoma. Am J Cardiol 2000;86:664-8.
12. Harris KM, Braverman AC, Gutierrez FR, et al. Transesophageal echocardiographic and clinical features of aortic intramural hematoma. J Thorac Cardiovasc Surg 1997;114:619-26.

13. Sawhney NS, DeMaria AN, Blanchard DG. Aortic intramural hematoma. Chest 2001;120:1340-6.

14. Evangelista A, Dominguez R, Sebastia C, et al. Prognostic value of clinical and morphologic findings in short-term evolution of aortic intramural hematoma. Eur Heart J 2004;25:81-7.

15. Vilacosta I, San Roman JA, Ferreiros J, et al. Natural history and serial morphology of aortic intramural hematoma: a novel variant of aortic dissection. Am Heart J 1997;134:495-507.

16. Keren A, Kim CB, Hu BS, et al. Accuracy of biplane and multiplane transesophageal echocardiography in diagnosis of typical acute aortic dissection and intramural hematoma. J Am Coll Cardiol 1996;28:627-36.

17. Kang DH, Song JK, Song MG, et al. Clinical and echocardiographic outcomes of aortic intramural hemorrhage compared with acute aortic dissection. Am J Cardiol 1998;81:202-6.

18. Song JK, Kim HS, Kang DH, et al. Different clinical features of aortic intramural hematoma versus dissection involving the ascending aorta. J Am Coll Cardiol 2001;37:1604-10.

19. Wilson SK, Hutchins GM. Aortic dissecting aneurysms causative factors in 204 subjects. Arch Pathol Lab Med 1982;106:175-80.

20. Shimizu H, Yoshino H, Udagawa H, et al. Prognosis of aortic intramural hemorrhage compared with classic aortic dissection. Am J Cardiol 2000;85:792-5.

21. Evangelista A, Mukherjee D, Mehta RH, et al. Acute Intramural hematoma of the aorta: A mystery in evolution. Circulation 2005;111:1063-70.

22. Hagan PG, Nienaber CA, Isselbacher E, et al. The international registry of acute aortic dissection (IRAD): new insights into and old disease. JAMA 2000;283:897-903.

23. Evangelista A, Czerny M, Nienaber C, et al. Interdisciplinary expert consensus on management of type $\mathrm{B}$ intramural haematoma and penetrating aortic ulcer. Eur J Cardiothorac Surg 2015;47:209-17.

24. Brinster DR. Endovascular repair of the descending thoracic aorta for penetrating atherosclerotic ulcer disease. J Card Surg 2009;24:203-8.

25. Hirst AE Jr, Barbour BH. Dissecting aneurysm with hemopericardium; report of a case with healing. N Engl J Med 1958;258:116-20. 
26. Vilacosta I, San Román JA, Aragoncillo P, et al. Penetrating atherosclerotic aortic ulcer: documentation by transesophageal echocardiography. J Am Coll Cardiol 1998;32:83-9.

27. Gutschow SE, Walker CM, Martínez-Jiménez S, et al. Emerging Concepts in Intramural Hematoma Imaging. Radiographics 2016;36:660-74.

28. Litmanovich D, Bankier A, Cantin L, et al. CT and MRI in Diseases of the Aorta. AJR Am J Roentgenol 2009;193:928-40.

29. Nathan DP, Boonn W, Lai E, et al. Presentation, complications, and natural history of penetrating atherosclerotic ulcer disease. J Vasc Surg 2012;55:10-5.

30. Evangelista A, Isselbacher EM, Bossone E, et al. Insights From the International Registry of Acute Aortic Dissection A 20-Year Experience of Collaborative Clinical Research. Circulation 2018;137:1846-60.

31. Mohr-Kahaly S, Erbel R, Kearney P, et al. Aortic Intramural Hemorrhage visualized by tranesophageal echocardiography: Findings and prognostic implications. J Am Coll Cardiol 1994;23:658-64.

32. Montgomery DH, Ververis JJ, McGorisk G, et al. Natural history of severe atheromatous disease of the thoracic aorta: A transesophageal echocardiographic study. J Am Coll Cardiol 1996;27:95-101.

33. Moral S, Cuéllar H, Avegliano G, et al. Clinical Implications of Focal Intimal Disruption in Patients With Type B Intramural Hematoma. J Am Coll Cardiol 2017;69:28-39.

34. Park GM, Ahn JM, Kim DH, et al. Distal aortic intramural hematoma: clinical importance of focal contrast enhancement on CT images. Radiology 2011;259:100-8.

35. Seitun S, Rossi UG, Cademartiri F, et al. MDCT findings of aortic branch artery pseudoaneurysms associated with type B intramural haematoma. Radiol Med 2012;117:789-803.

36. Williams DM, Cronin P, Dasika N, et al. Aortic branch artery pseudoaneurysms accompanying aortic dissection. Part I. Pseudoaneurysm anatomy. J Vasc Interv Radiol 2006; 17:765-71.

37. Wu MT, Wang YC, Huang YL, et al. Intramural blood pools accompanying aortic intramural hematoma: CT appearance and natural course. Radiology 2011;258:705-13.

38. Nienaber CA, Richartz BM, Rehders T, et al. Aortic intramural hematoma: natural history and predictive factors for complications. Heart 2004;90:372-4.

39. Sueyoshi E, Matsuoka Y, Sakamoto I, et al. Fate of intramural hematoma of the aorta: CT evaluation. J
Comput Assist Tomogr 1997;21:931-8.

40. Evangelista A. Aortic intramural haematoma: remarks and conclusions. Heart 2004;90:379-80.

41. Nishigami K, Tsuchiya T, Shono H, et al. Disappearance of aortic intramural hematoma and its significance to the prognosis. Circulation 2000;102:III243-7.

42. Choi SH, Choi SJ, Kim JH, et al. Useful CT findings for predicting the progression of aortic intramural hematoma to overt aortic dissection. J Comput Assist Tomogr 2001;25:295-9.

43. Ganaha F, Miller DC, Sugimoto K, et al. The prognosis of aortic intramural hematoma with and without penetrating atherosclerotic ulcer: a clinical and radiological analysis. Circulation 2002;106:342-8.

44. Sueyoshi E, Imada T, Sakamoto I, et al. Analysis of predictive factors for progression of type B aortic intramural hematoma with computed tomography. J Vasc Surg 2002;35:1179-83.

45. Mukherjee D, Evangelista A, Nienaber CA, et al. Implications of periaortic hematoma in patients with acute aortic dissection (from the International Registry of Acute Aortic Dissection). Am J Cardiol 2005;96:1734-8.

46. Kitai T, Kaji S, Yamamuro A, et al. Impact of new development of ulcer-like projection on clinical outcomes in patients with type $\mathrm{B}$ aortic dissection with closed and thrombosed false lumen. Circulation 2010;122:S74-80.

47. Sueyoshi E, Matsuoka Y, Imada T, et al. New development of an ulcerlike projection in aortic intramural hematoma: CT evaluation. Radiology 2002;224:536-41.

48. Evangelista A, Dominguez R, Sebastia C, et al. Long-term follow-up of aortic intramural hematoma. Predictors of outcome. Circulation 2003;108;583-9.

49. Moral S, Ballesteros E, Roque M, et al. Intimal disruption in type B aortic intramural hematoma. Does size matter? A systematic review and meta-analysis. Int J Cardiol 2018;269:298-303.

50. Ferro C, Rossi UG, Seitun S, et al. Aortic branch artery pseudoaneurysms associated with intramural hematoma: when and how to do endovascular embolization. Cardiovasc Intervent Radiol 2013;36:422-32.

51. Bischoff MS, Geisbüsch P, Peters AS, et al. Penetrating aortic ulcer: Defining risks and therapeutic strategies. Herz 2011;36:498-504.

52. Oderich GS, Kärkkäinen JM, Reed NR, et al. Penetrating Aortic Ulcer and Intramural Hematoma. Cardiovasc Intervent Radiol 2019;42:321-34.

53. Tittle SL, Lynch RJ, Cole PE, et al. Midterm follow-up of penetrating ulcer and intramural hematoma of the aorta. J 
Thorac Cardiovasc Surg 2002;123:1051-9.

54. Cho KR, Stanson AW, Potter DD, et al. Penetrating atherosclerotic ulcer of the descending thoracic aorta and arch. J Thorac Cardiovasc Surg 2004;127:1393-9.

55. Iyer VS, Mackenzie KS, Tse LW, et al. Early outcomes after elective and emergent endovascular repair of the thoracic aorta. J Vasc Surg 2006;43:677-83.

56. Scharrer-Pamler R, Kotsis T, Kapfer X, et al. Complications after endovascular treatment of thoracic aortic aneurysms. J Endovasc Ther 2003;10:711-8.

57. Patel HJ, Williams DM, Upchurch GR, et al. The challenge of associated intramural hematoma with endovascular repair for penetrating ulcers of the

Cite this article as: Evangelista A, Maldonado G, Moral S, Teixido-Tura G, Lopez A, Cuellar H, Rodriguez-Palomares J. Intramural hematoma and penetrating ulcer in the descending aorta: differences and similarities. Ann Cardiothorac Surg 2019;8(4):456-470. doi: 10.21037/acs.2019.07.05 descending thoracic aorta. J Vasc Surg 2010;51:829-35.

58. Jánosi RA, Gorla R, Tsagakis K, et al. Thoracic Endovascular Repair of Complicated Penetrating Aortic Ulcer: An 11-Year Single-Center Experience. J Endovasc Ther 2016;23:150-9.

59. Kaji S, Akasaka T, Katayama M, et al. Long-term prognosis of patients with type B aortic intramural hematoma. Circulation 2003;108:II307-11.

60. Song JM, Kang DH, Song JK, et al. Clinical significance of echo-free space detected by transesophageal echocardiography in patients with type B aortic intramural hematoma. Am J Cardiol 2002;89:548-51. 